\title{
General actions of hyperstructures and some applications
}

\author{
Jan Chvalina, Šárka Hošková-Mayerová and A. D. Nezhad
}

\begin{abstract}
The aim of this paper is to investigate useful generalizations of the classical concept of a quasi-automaton without outputs or a discrete dynamical system, which are also called actions of semigroups or groups on given phase sets. The paper contains also certain applications of presented concepts and examples from various areas of mathematics.
\end{abstract}

\section{Introduction}

The paper is devoted to investigation of a certain generalization of quasiautomata (called also automata without outputs), which are in fact discrete dynamical systems and to some of their applications. In section 2 and 3 we give some basic definitions and then, we consider three types of actions. In section 4 we present some applications. Moreover in section 5 there are described some applications of formerly investigated hyperstructures and corrected certain mistake from [11]. In connection with non-deterministic automata, or with multifunctions (relations) on algebraic structures and topological spaces seems to be natural to investigate actions of multistructures on sets of various objects. Some motivating factors come from the general system theory $[8,18]$; one illustrating example below is based on the concept of a general time system. In this connection in $[5,6]$ there are investigated various types of binary relations and hyperstructures.

Key Words: Action of a hyperstructure on a set, General n-hyperstructure, Transformation hypergroup, Fredholm integral operator, Ordinary and partial differential operator

2010 Mathematics Subject Classification: Primary 20N20; Secondary 37L99, 68Q70.

Received: August, 2011.

Revised: March, 2012

Accepted: March, 2012. 


\section{Preliminaries}

We use $[4,7,12]$ for terminology and notations which are not defined here. We suppose that the reader is familiar with some useful notation in hyperstructure theory and other related concepts. What follows now are some definitions and propositions in the theory of hyperstructure which we need for formulation of our results and in the proofs of our main results.

For an arbitrary $x$ from an ordered set $H$ we denote by $[x)_{\leq}=\{y \in H \mid x \leq y\}$ the upper end generated by $x$.

The following lemma is called Ends Lemma.

Lemma 2.1. $[2,19]$ Let $(H, \circ, \leq)$ be an ordered semigroup. Let $a \star b=[a \circ b)_{\leq}$ for any $a, b \in H$. The following conditions are equivalent:

1) For any pair $a, b \in H$ there exists a pair $c, d \in H$ such that $b \circ c \leq a$, $c \circ d \leq a$.

2) The hypergroupoid $(H, \star)$ associated with $(H, \circ, \leq)$ satisfies the associativity law and the reproduction axioms, i.e., $(H, \star)$ is a hypergroup.

Dually we can define the Beginnings Lemma:

Lemma 2.2. [2] Let $(H, \circ, \leq)$ be an ordered semigroup. Let $a \star b=(a \circ b]_{\leq}$ for any $a, b \in H$. The following conditions are equivalent:

1) For any pair $a, b \in H$ there exists a pair $c, d \in H$ such that $b \circ c \geq a$, $c \circ d \geq a$.

2) The hypergroupoid $(H, \star)$ associated with $(H, \circ, \leq)$ satisfies the associativity law and the reproduction axioms, i.e., $(H, \star)$ is a hypergroup.

Quasi-order hypergroups have been introduced and studied by J. Chvalina. The following definition can be found e.g. in $[4,19,20]$.

Definition 2.3. A hypergroup $(H, \star)$ such that the following conditions are satisfied:

1) $a \in a^{2}=a^{3}$ for any $a \in H$,

2) $a \star b=a^{2} \cup b^{2}$ for any pair $a, b \in H$ is called a quasi-order hypergroup. If moreover the unique square root condition:

3) $a, b \in H, a^{2}=b^{2}$ implies $a=b$

is satisfied then $(H, \star)$ is called an order hypergroup. 
Definition 2.4. [16] A hypergroup $(G, \star)$ is called a transposition hypergroup if it satisfies the transposition axiom: For all $a, b, c, d \in G$ the relation $b \backslash a \cap$ $c / d \neq \emptyset$ implies $a \star d \cap b \star c \neq \emptyset$. The sets $b \backslash a=\{x \in G \mid a \in b \star x\}, c / d=\{x \in$ $G \mid c \in x \star d\}$ are called left and right extensions, respectively.

Definition 2.5. $[12,13,14]$ Let $X$ be a set, $(G, \bullet)$ be a (semi)hypergroup and $\pi: X \times G \rightarrow X$ a mapping such that

$$
\pi(\pi(x, t), s) \in \pi(x, t \bullet s), \text { where } \pi(x, t \bullet s)=\{\pi(x, u) ; u \in t \bullet s)\}
$$

for each $x \in X, s, t \in G$. Then $(X, G, \pi)$ is called a discrete transformation (semi)hypergroup or an action of the (semi)hypergroup $G$ on the phase set $X$. The mapping $\pi$ is usually said to be simply an action. The condition (2.1) is called Generalized Mixed Associativity Condition, shortly GMAC.

\section{General hyperstructures}

Throughout this paper, the symbol $X, Y$ will denote two non-empty sets, where $P^{*}(X \cup Y)$ denotes the set of all non-empty subsets of $X \cup Y$.

A general hyperstructure is formed by two non-empty sets $X, Y$ together with a hyperoperation,

$$
*: X \times Y \longrightarrow P^{*}(X \cup Y), \quad(x, y) \mapsto x * y \subseteq(X \cup Y) \backslash \emptyset .
$$

Remark. A general hyperoperation $*: X \times Y \longrightarrow P^{*}(X \cup Y)$ yields a map of powersets determined by this hyperoperation. Thus the map $\otimes$ : $P^{*}(X) \times P^{*}(Y) \longrightarrow P^{*}(X \cup Y)$ is defined by $A \otimes B=\bigcup_{a \in A, b \in B} a * b$.

Conversely an general hyperoperation on $P^{*}(X) \times P^{*}(Y)$ yields a general hyperoperation on $X \times Y$, defined by $x * y=\{x\} \otimes\{y\}$.

In the above definition if $A \subseteq X, B \subseteq Y, x \in X, y \in Y$, then we define,

$$
\begin{gathered}
A * y=A *\{y\}=\bigcup_{a \in A} a * y, \quad x * B=\{x\} * B=\bigcup_{b \in B} x * b, \\
A \otimes B=\bigcup_{a \in A, b \in B} a * b .
\end{gathered}
$$

If $X=Y=H$, then we obtain the classical hyperstructure theory.

The concept of general hyperstructure with a hyperoperation which is a mapping $*: X \times Y \longrightarrow P^{*}(X \cup Y)$ mentioned above (used by A. Dehghan Nezhad and R. S. Hashemi, see [9]) allows straightforward generalization onto case of "hyperoperation of an arbitrary finite arity" in the following way: 
Definition 3.1. Let $n \in \mathbb{N}$ be an arbitrary positive integer, $n \geq 1$. Let $\left\{X_{k} ; k=1, \ldots, n\right\}$ be a system of non-empty sets. By a general $n$-hyperstructure we mean the pair $\left(\left\{X_{k} ; k=1, \ldots, n\right\}, *_{n}\right)$, where

$$
*_{n}: \prod_{k=1}^{n} X_{k} \rightarrow \mathcal{P}^{*}\left(\bigcup_{k=1}^{n} X_{k}\right)
$$

is a mapping assigning to any $n$-tuple $\left(x_{1}, \ldots, x_{n}\right) \in \prod_{k=1}^{n} X_{k}$ a non-empty subset $*_{n}\left(x_{1}, \ldots, x_{n}\right) \subset \bigcup_{k=1}^{n} X_{k}$.

Similarly as above, with this hyperoperation there is associated a mapping of power sets $\otimes_{n}: \prod_{k=1}^{n} \mathcal{P}^{*}\left(X_{k}\right) \rightarrow \mathcal{P}^{*}\left(\bigcup_{k=1}^{n} X_{k}\right)$ defined by $\otimes_{n}\left(A_{1}, \ldots, A_{n}\right)$ $=\bigcup\left\{*_{n}\left(x_{1}, \ldots, x_{n}\right) ;\left(x_{1}, \ldots, x_{n}\right) \in \prod_{k=1}^{n} A_{k}\right\}$. This construction is based on an idea of Nezhad and Hashemi [9] for $n=2$. Hyperstructures with $n$-ary hyperoperations are investigated among others in [21].

The results presented below are in a close connections with [7].

Example 3.2. Let $J \subset \mathbb{R}$ be an open interval, $\mathbb{C}^{n}(J)$ be the ring (with respect to usual addition and multiplication of functions) of all real functions $f: J \rightarrow \mathbb{R}$ with continuous derivatives up to the order $n \geq 0$ including. Denote

$$
L\left(p_{0}, p_{1}, \ldots, p_{n-1}\right): \mathbb{C}^{n}(J) \rightarrow \mathbb{C}^{n}(J)
$$

the linear differential operator defined by

$$
L\left(p_{0}, p_{1}, \ldots, p_{n-1}\right)(y)=\frac{\mathrm{d}^{n} y(x)}{\mathrm{d} x^{n}}+\sum_{s=0}^{n-1} p_{s}(x) \frac{\mathrm{d}^{s} y(x)}{\mathrm{d} x^{s}}
$$

where $y \in \mathbb{C}^{n}(J)$ and $p_{s} \in \mathbb{C}^{n}(J), s=0,1, \ldots, n-1$. In accordance with [1] we put

$$
\mathbb{L} \mathbb{A}_{n}(J)=\left\{L\left(p_{0}, \ldots, p_{n-1}\right) ; p_{k} \in \mathbb{C}^{n}(J)\right\} .
$$

Instead of $L\left(p_{1,0}, p_{1,1}, \ldots, p_{1, n-1}\right)$ we write $L\left(\vec{p}_{1}\right)$. We put $L\left(\vec{p}_{1}\right) \leq L\left(\vec{p}_{2}\right)$ whenever

$L\left(\vec{p}_{j}\right)=L\left(p_{j, 0}, \ldots, p_{j, n-1}\right), j=1,2, p_{1, s}(x) \leq p_{2, s}(x), s=0,1, \ldots, n-1$, $x \in J$ and $p_{1,0}(x) \equiv p_{2,0}(x)$. Defining

$$
*_{n}\left(L\left(\vec{p}_{1}\right), L\left(\vec{p}_{2}\right), \ldots, L\left(\vec{p}_{n}\right)\right)=\bigcup_{k=1}^{n}\left\{L(\vec{p}) \in \mathbb{L} \mathbb{A}_{k}(J) ; L\left(\vec{p}_{k}\right) \leq L(\vec{p})\right\}
$$


for any $n$-tuple $\left(L\left(\vec{p}_{1}\right), L\left(\vec{p}_{2}\right), \ldots, L\left(\vec{p}_{n}\right)\right) \in \prod_{k=1}^{n} \mathbb{L}_{\mathbb{A}_{k}}(J)$ we obtain that $\mathbb{L}(n)=\left(\left\{\mathbb{L} \mathbb{A}_{k}(J) ; k=1,2, \ldots, n\right\}, *_{n}\right)$ is a general $n$-hyperstructure.

Of course, $\mathbb{L} \mathbb{A}_{1}(J)$ is the set of all first-order linear differential operators of the form $L\left(p_{0}\right)(y)=y^{\prime}(x)+p_{0}(x) y$, where $p_{0} \in \mathbb{C}(J)$ and $y \in \mathbb{C}^{1}(J)$. Evidently $\mathbb{L A}_{j}(J) \cap \mathbb{L} \mathbb{A}_{k}(J)=\emptyset$ whenever $j \neq k$.

It is to be noted that if $k, m \in\{1,2, \ldots, n\}$ are fixed different integers then setting $X=\mathbb{L} \mathbb{A}_{k}(J), Y=\mathbb{L} \mathbb{A}_{m}(J)$ we obtain from the above construction an example of a general hyperstructure in sense of Nezhad and Hashemi [9]. If, moreover $X=Y=\mathbb{L} \mathbb{A}_{n}(J)$ then the resulting general hyperstructure is an order hypergroup of linear differential $n$-order operators in the sense of e.g. [1].

Definition 3.3. Let $\mathbb{G}_{1}(n)=\left(\left\{X_{k} ; k=1, \ldots, n\right\}, *_{n}\right), \mathbb{G}_{2}(n)=\left(\left\{Y_{k} ; k=\right.\right.$ $\left.1, \ldots, n\}, \bullet_{n}\right)$, be a pair of general $n$-hyperstructures. By a good homomorphism $H: \mathbb{G}_{1}(n) \rightarrow \mathbb{G}_{2}(n)$ we mean any system of mappings $H=\left\{h_{k}: X_{k} \rightarrow\right.$ $\left.Y_{k}\right\}$ such that the following diagram is commutative:

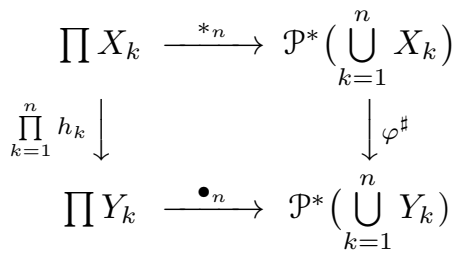

Here $\prod_{k=1}^{n} h_{k}\left(x_{1}, x_{2}, \ldots, x_{n}\right)=\left(h_{1}\left(x_{1}\right), h_{2}\left(x_{2}\right), \ldots, h_{n}\left(x_{n}\right)\right)$ for any $n$-tuple $\left(x_{1}, x_{2}, \ldots, x_{n}\right) \in \prod_{k=1}^{n} X_{k}$ and $\varphi^{\sharp}: \mathcal{P}^{*}\left(\bigcup_{k=1}^{n} X_{k}\right) \rightarrow \mathcal{P}^{*}\left(\bigcup_{k=1}^{n} Y_{k}\right)$ is the lifting of a mapping $\varphi$ : $\bigcup_{k=1}^{n} X_{k} \rightarrow \bigcup_{k=1}^{n} Y_{k}$ defined by the induction. For $x \in X_{1}$ we put $\varphi(x)=h_{1}(x)$. Suppose $\varphi: \bigcup_{j=1}^{k} X_{j} \rightarrow \bigcup_{j=1}^{k} Y_{j}$ is well-defined. Then for any $x \in X_{k+1} \backslash \bigcup_{j=1}^{k} X_{j}$ we put $\varphi(x)=h_{k+1}(x)$.

As a certain generalization of the general $n$-hyperstructure from Example 3.2 we will construct the following structure:

Example 3.4. Consider a system of pairwise disjoint ordered sets $\left(X_{k}, \leq_{k}\right)$, $k=1,2, \ldots, n$, (where $n$ is a given positive integer) and for $x \in X_{k}$ let us denote $[x)_{k}=\left\{y \in X_{k} ; x \leq_{k} y\right\}$, i.e. $[x)_{k}$ is the principal end generated by the element $x$ within the ordered set $\left(X_{k}, \leq_{k}\right)$. Further, put 


$$
*_{n}\left(x_{1}, x_{2}, \ldots, x_{n}\right)=\bigcup_{k=1}^{n}\left[x_{k}\right)_{k}
$$

for any $n$-tupple $*_{n}\left(x_{1}, x_{2}, \ldots, x_{n}\right) \in \prod_{k=1}^{n} X_{k}$. Then $*\left(x_{1}, x_{2}, \ldots, x_{n}\right) \subseteq \bigcup_{k=1}^{n} X_{k}$, thus $\mathbb{G}(n)=\left(\left\{X_{k} ; k=1, \ldots, n\right\}, *\right)$ is a general $n$-hyperstructure in the sense of the above definition. If $\mathbb{H}(n)=\left(\left\{Y_{k} ; k=1, \ldots, n\right\}, \bullet_{n}\right)$ is a general $n$ hyperstructure such that $\left(Y_{k}, \preceq_{k}\right), k=1, \ldots, n$ are pairwise disjoint ordered sets and

$$
\bullet_{n}\left(y_{1}, y_{2}, \ldots, y_{n}\right)=\bigcup_{k=1}^{n}\left[y_{k}\right)_{k} \subseteq \bigcup_{k=1}^{n} Y_{k}
$$

for any $n$-tuple $\left(y_{1}, y_{2}, \ldots, y_{n}\right) \in \prod_{k=1}^{n} Y_{k}$ we consider a system $h_{k}:\left(X_{k}, \leq_{k}\right)$ $\rightarrow\left(Y_{k}, \preceq_{k}\right), k=1, \ldots, n$, of strongly isotone mappings, i.e. for any $x \in$ $X_{k}$ there holds $h_{k}\left(\left[x_{k}\right)_{k}\right)=\left[h_{k}\left(x_{k}\right)\right)_{k}, k=1, \ldots, n$. Then denoting $H=$ $\left\{h_{k}: X_{k} \rightarrow Y_{k} ; k=1, \ldots, n\right\}$ we obtain that $H$ is a good homomorphism of the general $n$-hyperstructure $\mathbb{G}(n)$ into the general $n$-hyperstructure $\mathbb{H}(n)$. Indeed, consider an arbitrary $n$-tuple $\left(x_{1}, x_{2}, \ldots, x_{k}\right) \in \prod_{k=1}^{n} X_{n}$. As above denote by $\varphi: \mathcal{P}^{*}\left(\bigcup_{k=1}^{n} X_{k}\right) \rightarrow \mathcal{P}^{*}\left(\bigcup_{k=1}^{n} Y_{k}\right)$ the lifting of the mapping $\varphi: \bigcup_{k=1}^{n} X_{k}$ $\rightarrow \bigcup_{k=1}^{n} Y_{k}$ induced by the system $\left\{h_{k}: X_{k} \rightarrow Y_{k} ; k=1, \ldots, n\right\}$-here in such a way that $\varphi \mid X_{k}=h_{k}$. Then for any $n$-tupple $\left(x_{1}, x_{2}, \ldots, x_{n}\right) \in \prod_{k=1}^{n} X_{k}$ we have

$$
\begin{aligned}
& \varphi\left(*_{n}\left(x_{1}, x_{2}, \ldots, x_{n}\right)\right)=\varphi\left(\bigcup_{k=1}^{n}\left[x_{k}\right)_{k}\right)=\bigcup_{k=1}^{n} \varphi\left(\left[x_{k}\right)_{k}\right)=\bigcup_{k=1}^{n} h_{k}\left(\left[x_{k}\right)_{k}\right) \\
& =\bigcup_{k=1}^{n}\left[h_{k}\left(x_{k}\right)_{k}\right)=\bullet_{n}\left(h_{1}\left(x_{1}\right), \ldots, h_{n}\left(x_{n}\right)\right)=\bullet_{n}\left(\left(\prod_{k=1}^{n} h_{k}\right)\left(x_{1}, x_{2}, \ldots, x_{n}\right)\right),
\end{aligned}
$$

i.e. $\varphi \circ *_{n}=\bullet_{n} \circ \prod_{k=1}^{n} h_{k}$, thus the diagram D2 is commutative. 


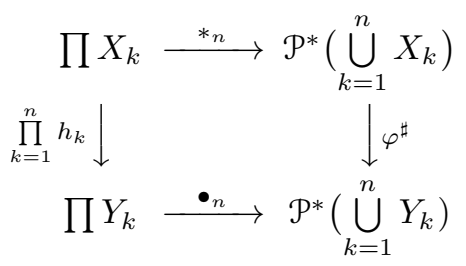

From the above example there follows immediately the following assertion.

Proposition 3.5. Let $\left(X_{k}, \leq_{k}\right), k=1, \ldots, n,\left(Y_{k}, \preceq_{k}\right), k=1, \ldots, n$, be two collections of pairwise disjoint ordered sets and $\mathbb{G}(n), \mathbb{H}(n)$ be corresponding $n$-general hyperstructures. Suppose $\left(X_{k}, \leq_{k}\right) \cong\left(Y_{k}, \preceq_{k}\right)$ for each $k=1, \ldots, n$ and $h_{k}:\left(X_{k}, \leq_{k}\right) \rightarrow\left(Y_{k}, \preceq_{k}\right)$ are corresponding order-isomorphisms. Then we have $\mathbb{G}(n) \cong \mathbb{H}(n)$.

The following text is a generalization of e.g. [3]. Suppose $u_{1}, \ldots, u_{n} \in$ $\mathbb{C}^{n}(J)$ is a linearly independent system of functions. Denote by $V\left(u_{1}, \ldots, u_{n}\right)$ the $n$-dimensional vector space generated by the base $u_{1}, \ldots, u_{n}$, i.e.

$$
V\left(u_{1}, \ldots, u_{n}\right)=\left\{\sum_{k=1}^{n} c_{k} u_{k} ; c_{k} \in \mathbb{R}, k=1, \ldots, n\right\} .
$$

The system $u_{1}, \ldots, u_{n}$ can be considered as a fundamental system of solutions of a differential equation

$$
y^{(n)}(x)+\sum_{k=0}^{n-1} p_{k}(x) y^{(k)}(x)=0
$$

where

$$
p_{k}(x)=\frac{D_{k}\left[u_{1}, \ldots, u_{n}\right]}{W\left[u_{1}, \ldots, u_{n}\right]}, \quad k=1, \ldots, n-1 .
$$

Here, $W\left[u_{1}, \ldots, u_{n}\right]$ is the Wronski determinant of the system and $D_{k}\left[u_{1}, \ldots, u_{n}\right]$ are corresponding subdeterminants of the determinant

$$
W\left[y_{1}, \ldots, y_{n}\right]=\left|\begin{array}{ccccc}
u_{1} & u_{2} & \ldots & u_{n} & y \\
u_{1}^{\prime} & u_{2}^{\prime} & \ldots & u_{n}^{\prime} & y^{\prime} \\
\vdots & \vdots & & \vdots & \\
u_{1}^{(n)} & u_{2}^{(n)} & \ldots & u_{n}^{(n)} & y^{(n)}
\end{array}\right|
$$

It has been mentioned in papers contained in References that one of significant result of the general theory of linear differential homogeneous equations is the fact that there is one-to-one correspondence between the system $\mathbb{L}_{\mathbb{A}_{n}}(J)$ of all linear ordinary differential operators of the form $(3.1)$ and 
the system $\mathbb{V} \mathbb{A}_{n}(J)$ of solution spaces of corresponding differential equations $L\left(p_{0}, \ldots, p_{n-1}\right) y=0, L\left(p_{0}, \ldots, p_{n-1}\right) \in \mathbb{L} \mathbb{A}_{n}(J)$. So, in what follows we will suppose that $\mathbb{L}_{k}(J)$ is the system of $n$-th order linear ordinary differential operators $L\left(p_{0}, \ldots, p_{k-1}\right)$, with $p_{0}(x)>0$ for all $x \in J, k=1,2, \ldots, n$ and $\mathbb{V A}_{n}(J)$ is the corresponding system of solution spaces of differential equations $L\left(p_{0}, \ldots, p_{k-1}\right) y=0$. Using the following specification of the binary operation considered in papers [1] and elsewhere we turn out the system $\mathbb{V} \mathbb{A}_{n}(J)$, $k=1,2, \ldots, n$ into a noncommutative group. In detail, for $L\left(p_{0}, \ldots, p_{k-1}\right)$, $L\left(q_{0}, \ldots, q_{k-1}\right) \in \mathbb{L} \mathbb{A}_{k}(J)$ we define for $x \in J, j=1,2, \ldots, k-1$

$$
L\left(p_{0}, \ldots, p_{k-1}\right) \cdot L\left(q_{0}, \ldots, q_{k-1}\right)=L\left(\varphi_{0}, \ldots, \varphi_{k-1}\right),
$$

where $\varphi_{0}(x)=p_{0}(x) q_{0}(x), \varphi_{j}(x)=p_{0}(x) q_{j}(x)+p_{j}(x)$. Then we obtain that $\mathbb{V A}_{n}(J)$ is a noncommutative group for $k=1,2, \ldots, n$.

Now using the just defined operation we can endow the system $\mathbb{V} \mathbb{A}_{n}(J)$ by corresponding binary operation in this way:

For an arbitrary pair of spaces $V\left(u_{1}, \ldots, u_{k}\right), V\left(v_{1}, \ldots, v_{k}\right) \in \mathbb{V} \mathbb{A}_{n}(J)$, there are uniquely determined operators

$L\left(p_{0}, \ldots, p_{k-1}\right)=\Phi_{k}^{-1}\left(V\left(u_{1}, \ldots, u_{k}\right)\right), L\left(q_{0}, \ldots, q_{k-1}\right)=\Phi_{k}^{-1}\left(V\left(v_{1}, \ldots, v_{k}\right)\right)$, $k=1,2, \ldots, n$, where $\Phi_{k}: \mathbb{L} \mathbb{A}_{k}(J) \rightarrow \mathbb{V A}_{k}(J)$ are the above mentioned bijections. Defining

$$
V\left(u_{1}, \ldots, u_{k}\right) \cdot V\left(v_{1}, \ldots, v_{k}\right)=\Phi_{k}\left(L\left(p_{0}, \ldots, p_{k-1}\right) \cdot L\left(q_{0}, \ldots, q_{k-1}\right)\right),
$$

we obtain $\mathbb{V} \mathbb{A}_{k}(J)$ is a noncommutative group, thus $\Phi_{k}: \mathbb{L}_{k}(J) \rightarrow \mathbb{V A}_{k}(J)$ is a group-isomorphism, $k=1,2, \ldots, n$. Notice, that for any pair of different integers $k, m \in \mathbb{N}$ we have $\mathbb{V A}_{k}(J) \cap \mathbb{V} \mathbb{A}_{m}(J) \neq \emptyset$ as well as $\mathbb{L} \mathbb{A}_{k}(J) \cap \mathbb{L} \mathbb{A}_{m}(J) \neq$ $\emptyset$.

Definition 3.6. Let $\mathbb{G}(n)=\left(\prod_{k=1}^{n} X_{k}, *_{n}, \mathcal{P}^{*}\left(\bigcup_{k=1}^{n} X_{k}\right)\right), \mathbb{H}(n)$ $=\left(\prod_{k=1}^{n} Y_{k}, \bullet_{n}, \mathcal{P}^{*}\left(\bigcup_{k=1}^{n} X_{k}\right)\right)$ be general $n$-hyperstructures, $F=\left\{f_{k}: X_{k} \rightarrow\right.$ $\left.Y_{k} ; k=1,2, \ldots, n\right\}$ be the system of mappings satisfying the conditions

$$
\text { - }_{n}\left(y_{1}, \ldots, y_{n}\right)=\varphi\left(*_{n}\left(f_{1}^{-1}\left(y_{1}\right), \ldots, f_{n}^{-1}\left(y_{1}\right)\right)\right) \subseteq \bigcup_{k=1}^{n} Y_{k}
$$

where $\varphi$ is determined by $F$ (i.e. $\varphi(x)=f_{1}(x)$ for $x \in X_{1}$ and supposing $\varphi(x)$ is defined for $x \in X_{j}$ then $\varphi(x)=f_{j+1}(x)$ for $\left.x \in X_{j+1} \backslash \bigcup_{m=1}^{j} X_{m}\right)$. Then the hyperoperation $\bullet_{n}$ is termed as the hyperoperation associated with the hyperoperation $*_{n}$. 
Theorem 3.7. Let $n \in \mathbb{N}$ be a positive integer, $J \subseteq \mathbb{R}$ be an open interval. If

$$
\mathbb{L}(J ; n)=\left(\prod_{k=1}^{n} \mathbb{L} \mathbb{A}_{k}(J), *_{n}, \mathcal{P}^{*}\left(\bigcup_{k=1}^{n} \mathbb{L} \mathbb{A}_{k}(J)\right)\right)
$$

is the general $n$-hyperstructure of ordinary linear differential operators and

$$
\mathbb{S}(J ; n)=\left(\prod_{k=1}^{n} \mathbb{V} \mathbb{A}_{k}(J), \bullet_{n}, \mathcal{P}^{*}\left(\bigcup_{k=1}^{n} \mathbb{V} \mathbb{A}_{k}(J)\right)\right)
$$

is the general n-hyperstructure of solution spaces of linear ordinary homogeneous differential equations associated with $\mathbb{L}(J ; n)$, then we have

$$
\mathbb{L}(J ; n) \cong \mathbb{S}(J ; n),
$$

i.e. in the commutative diagram

$$
\begin{aligned}
& \prod_{k=1}^{n} \mathbb{L} \mathbb{A}_{k}(J) \stackrel{*_{n}}{\longrightarrow} \mathcal{P} *\left(\bigcup_{k=1}^{n} \mathbb{L} \mathbb{A}_{k}(J)\right) \\
& \prod_{k=1}^{n} \Phi_{k} \downarrow \\
& \prod_{k=1}^{n} \mathbb{V} \mathbb{A}_{k}(J) \stackrel{\bullet_{n}}{\longrightarrow} \mathcal{P}^{*}\left(\bigcup_{k=1}^{n} \mathbb{V} \mathbb{A}_{k}(J)\right)
\end{aligned}
$$

arrows $\prod_{k=1}^{n} \Phi_{k}, \varphi^{\sharp}$ are bijections.

Proof. By [1] we have $\Phi_{k}: \mathbb{L}_{k}(J) \rightarrow \mathbb{V A}_{k}(J)$ is a group-isomorphism for any $k=1,2, \ldots, n$ thus $\prod_{k=1}^{n} \Phi_{k}: \prod_{k=1}^{n} \mathbb{L} \mathbb{A}_{k}(J) \rightarrow \prod_{k=1}^{n} \mathbb{V} \mathbb{A}_{k}(J)$ is a bijection. Since $\left\{\mathbb{L} \mathbb{A}_{k}(J) ; k=1,2, \ldots, n\right\},\left\{\mathbb{V} \mathbb{A}_{k}(J) ; k=1,2, \ldots, n\right\}$ are pairwise disjoint families we have that the mapping $\varphi: \bigcup_{k=1}^{n} \mathbb{L} \mathbb{A}_{k}(J) \rightarrow \bigcup_{k=1}^{n} \mathbb{V} \mathbb{A}_{k}(J)$ such that $\varphi \mid \mathbb{L} \mathbb{A}_{k}(J)=\Phi_{k}$ is a well-defined bijection hence the bijection $\mathcal{P}^{*}\left(\bigcup_{k=1}^{n} \mathbb{L}_{\mathbb{A}_{k}}(J)\right) \rightarrow$ $\mathcal{P}^{*}\left(\bigcup_{k=1}^{n} \mathbb{V} \mathbb{A}_{k}(J)\right)$ is also well-defined.

Now, for an arbitrary $n$-tuple $\left(L_{1}, \cdots, L_{n}\right) \in \prod_{k=1}^{n} \mathbb{L}_{\mathbb{A}_{k}}(J)$ we obtain that $\bullet_{n}\left(\left(\prod_{k=1}^{n} \Phi_{k}\right)\left(L_{1}, \cdots, L_{n}\right)\right)=\bullet_{n}\left(\Phi_{1}\left(L_{1}\right), \cdots, \Phi_{n}\left(L_{n}\right)\right)=\bullet_{n}\left(V_{1}, \cdots, V_{n}\right)=$ $=\varphi\left(*_{n}\left(\Phi_{1}^{-1}\left(V_{1}\right), \cdots, \Phi_{n}^{-1}\left(V_{n}\right)\right)\right)=\varphi^{\sharp}\left(*_{n}\left(L_{1}, \cdots, L_{n}\right)\right)$, 
since the hyperoperation "• $n$ " is associated with the hyperoperation " $*_{n}$ " . Therefore the diagram D3 in Theorem 3.7 is commutative.

Let $\left\{\left(S_{k}, \cdot \leq_{k}\right) ; k=1,2, \cdots, n\right\}$ be a system of quasi-ordered semigroups. Define a mapping $\odot_{n}: \prod_{k=1}^{n} S_{k} \rightarrow \mathcal{P}^{*}\left(\bigcup_{k=1}^{n} S_{k}\right)$ by the rule

$$
\odot_{n}\left(x_{1}, \ldots, x_{n}\right)=\bigcup_{k=1}^{n}\left[x_{k}^{2}\right)_{\leq_{k}}
$$

for any $n$-tuple $\left(x_{1}, \ldots, x_{n}\right) \in \prod_{k=1}^{n} S_{k}$. Then the general $n$-hyperstructure is called the general n-hyperstructure determined by the Ends Lemma or shortly EL-determined general n-hyperstructure.

Corollary of Theorem 3.7 Let $n \in \mathbb{N}$ be an integer, $J \subseteq \mathbb{R}$ be an open interval. Let $\mathbb{L}^{E L}(J ; n)=\left(\prod_{k=1}^{n} \mathbb{L} \mathbb{A}_{k}(J), *_{n}, \mathcal{P}^{*}\left(\bigcup_{k=1}^{n} \mathbb{L} \mathbb{A}_{k}(J)\right)\right)$ be the EL-determined general $n$-hyperstructure of all linear ordinary differential operators of all orders $k=1,2, \cdots, n$.

Let $\mathbb{S}^{E L}(J ; n)=\left(\prod_{k=1}^{n} \mathbb{V} \mathbb{A}_{k}(J), *_{n}, \mathcal{P}^{*}\left(\bigcup_{k=1}^{n} \mathbb{V A}_{k}(J)\right)\right)$ be the EL-determined general $n$-hyperstructure of solutions of homogeneous linear ordinary differential equations $L y=0, L \in \bigcup_{k=1}^{n} \mathbb{L} \mathbb{A}_{k}(J)$. Then $\mathbb{L}^{E L}(J ; n) \cong \mathbb{S}^{E L}(J ; n)$.

In the above construction we can use a finite sequence of positive integers $\left\{m_{1}, m_{2}, \ldots, m_{n}\right\}$ and then define the $n$-hyperoperation $\odot_{n}\left(x_{1}, \ldots, x_{n}\right)=$ $\bigcup_{k=1}^{n}\left[x_{k}^{m_{k}}\right)_{\leq_{k}}$ for any $n$-tuple $\left(x_{1}, \ldots, x_{n}\right) \in \prod_{k=1}^{n} S_{k}$.

\section{General R-hyperstructures (or L-hyperstruc- tures)}

Definition 4.1. A general Right hyperstructure (or Left hyperstructure) is the quadruple $\left(X, Y, \mathcal{P}^{*}(X), *_{R}\right)$ or $\left(X, Y, \mathcal{P}^{*}(X), *_{L}\right)$, shortly general $R$-hyperstructure or general L-hyperstructure, where $X, Y \neq \emptyset$ and

$$
\begin{aligned}
& *_{R}: X \times Y \longrightarrow P^{*}(X) \quad \text { or } \quad *_{L}: X \times Y \longrightarrow P^{*}(Y) \\
& (x, y) \mapsto x *_{R} y \subseteq X, \quad(x, y) \mapsto x *_{L} y \subseteq Y .
\end{aligned}
$$

The set of points $Y_{R} x=\left\{x *_{R} y: y \in Y\right\}$ that can be reached from a given point $x \in X$ by the R-hyperoperation of two non-empty sets $X, Y$, is called the R-hyperorbit of $x$. 
Example 4.2. Let $X \neq \emptyset$ be an arbitrary set, $f: X \rightarrow X$ be a mapping, i.e. the pair $(X, f)$ is a monounary algebra. Put $Y=\mathbb{N}$ (the set of all positive integers) and define $*_{R}^{f}: X \times Y \rightarrow \mathcal{P}^{*}(X)$ by the rule $x *_{R}^{f} n=\left\{f^{k}(x) ; k \in \mathbb{N}, n \leq\right.$ $k\}$. Then the quadruple $\left(X, Y, \mathcal{P}^{*}(X), *_{R}^{f}\right)$ is a general Right hyperstructure, i.e. R-hyperstructure. (Here, $f^{k}$ is the $k$-th iteration of $f$ ).

Example 4.3. Let $T$ be a linearly ordered set (i.e. a chain) with the least element. Then $T$ is called a time scale or time axis. Suppose $A \neq \emptyset \neq B$ are arbitrary sets and $S$ is a binary relation between sets of mappings (impulses) $A^{T}, B^{T}$, i.e. $S \subset A^{T} \times B^{T}$. Then the triad $\left(A^{T}, B^{T}, S\right)$ is called a general time system with input space $A^{T}$, the output space $B^{T}$ and with input-output relation (or the transition relation) $S$-cf.[18]. Now, denote $X=A^{T}, Y=B^{T}$ and define $*_{L}^{S}: X \times Y \rightarrow \mathcal{P}^{*}(Y)$ by $x *_{L}^{S} y=S(x)=\{u \in Y ; x S u\}$ for any pair of time-impulses $x: T \rightarrow A, y: T \rightarrow B$. Then we obtain the quadruple $\left(X, Y, \mathcal{P}^{*}(X), *_{L}^{S}\right)$ which is a general Left hyperstructure, i.e. a general Lhyperstructure.

\subsection{L-hyperaction (or R-hyperaction) of a hyperstructure on a non-empty set}

In this section, we give two new definitions. Let us make our point clear with an example.

Definition 4.4. Let $(G, \star)$ be a hyperstructure and $X$ be a non-empty set. A generalized L-hyperaction of $\mathrm{G}$ on $\mathrm{X}$ is a L-hyperoperation $\psi: G \times X \longrightarrow$ $\mathcal{P}^{*}(X)$ such that the following axioms are satisfied:

1) For all $g, h \in G$ and $x \in X, \psi(g \star h, x) \subseteq \psi(g, \psi(h, x))$,

2) For all $g \in G, \psi(g, X)=X$.

For any $g \in G$ and $A \subseteq X, \psi(g, A)=\bigcup_{x \in A} \psi(g, x)$, also for any $x \in X$ and $B \subseteq$ $G, \psi(B, x)=\bigcup_{b \in B} \psi(b, x)$. If in the axiom 1) of definition the equality holds, the corresponding generalized L-hyperaction is called strong. The generalized R-hyperaction (eventually strong) is defined dually.

Example 4.5. Consider the set of $n \times n$ symmetric, positive definite matrices, $S P D(n)$. The group $G L(n)=G L(n, R)$ hyperacts on $S P D(n)$ as follows; for all $A \in G L(n)$ and all $S \in S P D(n), A * S=\left\{A S A^{T}, A^{T} S A\right\}$.

It is easily checked that $A S A^{T}$ is in $S P D(n)$ if $S$ is in $S P D(n)$. For every $S P D$ matrix $S$, can be written as $S=A A^{T}$, for some invertible matrix $A$. 
Proposition 4.6. The map $\psi$ is a generalized action of $\operatorname{Sym}_{e}(G)$ on $G$.

Proof. We have; $\psi(s, \psi(r, g))=\psi(s, r(<g>)))=\bigcup_{i \in Z} \psi\left(s, r\left(g^{i}\right)\right)$

$=\bigcup_{i \in Z} s\left(<r\left(g^{i}\right)>\right)=\left\{s\left(\left(r\left(g^{i}\right)\right)^{j}\right) \mid i, j \in Z\right\}$ and $\psi(s r, g)=s r(<g>)=$ $\left\{s r\left(g^{i}\right) \mid i \in Z\right\}=\left\{s\left(r\left(g^{i}\right)\right) \mid i \in Z\right\}$. This shows that $\psi(s r, g) \subseteq \psi(s, \psi(r, g))$.

On the other hand, for all $r \in \operatorname{Sym}_{e}(G)$, we have

$$
\psi(r, G)=\bigcup_{g \in G} \psi(r, g)=\bigcup_{g \in G} r(<g>)=G,
$$

which completes the proof.

In the following proposition we will consider the classical interval binary hyperoperation on a linearly ordered group, see [15]. In detail if $(G, \cdot, \leq)$ is a linearly ordered group then we define a binary hyperoperation $*: G \times G \rightarrow$ $\mathcal{P}^{*}(G)$ by

$$
\begin{aligned}
a * b & =[\min \{a, b\})_{\leq} \cap(\max \{a, b\}]_{\leq}=[\min \{a, b\}, \max \{a, b\}]_{\leq} \\
& =\{x \in G ; \min \{a, b\} \leq x \leq \max \{a, b\}\}
\end{aligned}
$$

(which is a closed interval) where $\min \{a, b\}, \max \{a, b\}$ is the least element, the greatest element of the set $\{a, b\}$, respectively. It is easy to verify that the obtained hypergroupoid $(G, *)$ is an extensive commutative hypergroup. This hypergroup we obtain even in the case if we restrict ourselves onto the set $G_{+}$ of all positive elements of the linearly ordered group $(G, \cdot, \leq$ ), (cf. the proof of Proposition 4.7).

Proposition 4.7. Let $(G, \cdot, \leq)$ be a linearly ordered group, $G_{+}$be its subset of all positive elements (i.e. the positive cone) endowed with the interval binary hyperoperation "* $L$ ". Define a mapping $\psi_{G}: G_{+} \times G \rightarrow \mathcal{P}^{*}(G)$ by

$$
\psi_{G}(a, b)=(a+b]_{\leq}=\{x \in G ; x \leq a+b\}
$$

for all pairs $(a, b) \in G_{+} \times G$. Then the quadruple $\left(G_{+}, G, \mathcal{P}^{*}(G), \psi_{G}\right)$ is the generalized L-hyperoperation of the commutative extensive hypergroup $\left(G, *_{L}\right)$ on the group $(G,+, \leq)$.

Proof. For any pair $(a, b) \in G_{+} \times G_{+}$we have

$$
\{a, b\} \subseteq[\min \{a, b\}, \max \{a, b\}]_{\leq}=a *_{L} b=b *_{L} a,
$$


thus $\left(G_{+}, *_{L}\right)$ is a commutative extensive hypergroupoid. Further, consider an arbitrary triad $a, b, c \in G_{+}$. Without loss of generality we can suppose $a \leq b$. If $a=c$ or $b=c$ then evidently

$$
\left(a *_{L} b\right) *_{L} c=[a, b]_{\leq}=a *_{L}\left(b *_{L} c\right)
$$

so, suppose $a<b, a \neq c \neq b$. Three cases are possible: (i) $c<a$, (ii) $a<$ $c<b, \quad$ (iii) $b<c$. In the first case (i) we have

$$
\begin{aligned}
\left(a *_{L} b\right) *_{L} c=[a, b]_{\leq} *_{L} c & =\bigcup_{x \in[a, b]_{\leq}} x *_{L} c=\bigcup_{x \in[a, b]_{\leq}}[c, x]_{\leq}=[c, b]_{\leq} \\
=[c, a]_{\leq} \cup[a, b]_{\leq} & =\bigcup_{x \in[c, b]_{\leq}} a *_{L} x=a *_{L}[c, b]_{\leq}=a *_{L}\left(b *_{L} c\right) .
\end{aligned}
$$

In the case (ii) we have

$$
\left(a *_{L} b\right) *_{L} c=\bigcup_{x \in[a, b]_{\leq}}[x, c]_{\leq}=[a, b]_{\leq}=\bigcup_{x \in[c, b]_{\leq}}[a, x]_{\leq}=a *_{L}\left(b *_{L} c\right) .
$$

In the case (iii) we obtain

$$
\left(a *_{L} b\right) *_{L} c=\bigcup_{x \in[a, b]_{\leq}}[x, c]_{\leq}=[a, c]=\bigcup_{x \in[b, c]_{\leq}}[a, x]_{\leq}=a *_{L}[b, c]_{\leq}=a *_{L}\left(b *_{L} c\right),
$$

hence the hypergroupoid $\left(G, *_{L}\right)$ is associative, thus it is a semihypergroup.

Since

$$
a *_{L} G_{+}=\bigcup_{x \in G_{+}}\left(a *_{L} x\right)=\bigcup_{x \in G_{+}}[a, x]_{\leq}=G_{+}
$$

we have $\left(G, *_{L}\right)$ is an extensive commutative hypergroup. It remains to show that conditions 1), 2) from Definition 4.4 are satisfied. So, let $g, h \in G_{+}$be elements such that $g<h, x \in G$. Then

$$
\begin{gathered}
\psi_{G}\left(g *_{L} h, x\right)=\psi_{G}\left([g, h]_{\leq}, x\right)=\bigcup_{t \in[g, h]_{\leq}} \psi_{G}(t, x)=\bigcup_{t \in[g, h]_{\leq}}(t+x]_{\leq}=(h+x]_{\leq}, \\
\psi_{G}(g, \psi(h, x))=\psi_{G}\left(g,(h+x]_{\leq}\right)=\bigcup_{u \in(h+x]_{\leq}} \psi_{G}(g, u)=[g+h+x)_{\leq} .
\end{gathered}
$$

Since $0 \leq g$ we have $h+x \leq g+h+x$ and

$$
\psi_{G}\left(g *_{L} h, x\right)=(h+x]_{\leq} \subseteq[g+h+x)_{\leq}=\psi_{G}(g, \psi(h, x)) .
$$

Further for any $g \in G_{+}$there holds $\psi_{G}(g, G)=\bigcup_{x \in G} \psi_{G}(g, x)=\bigcup_{x \in G}(g+x]_{\leq}=$ $G$. Therefore conditions 1), 2) are satisfied, thus the proof is complete. 
Definition 4.8. Let $\mathrm{X}$ be a set, $(G, \star)$ be a semihypergroup and $\psi: G \times X \longrightarrow$ $\mathcal{P}^{*}(X)$ be a mapping such that

$$
\psi(h, \psi(g, x)) \subseteq \psi(g \star h, x) \text { where } \psi(g \star h, x)=\{\psi(t, x) \mid t \in g \star h\}
$$

for each $x \in X, g, h \in G$ then $(X, G, \psi)$ is called a generalized transformation semihypergroup.

This structure type is a generalization considered in $[10,11,13]$.

\subsubsection{Homomorphism of transformation semihypergroups}

Definition 4.9. Let $(X, G, \psi),(Y, H, \omega)$ be two generalized transformation semihypergroups (GTS). A pair of mappings $\Phi=[\mu, \varphi]$ such that $\mu: G \rightarrow H$ is a homomorphism of semihypergroups and $\varphi: X \rightarrow Y$ is a mapping, is said to be a homomorphism of $\operatorname{GTS}(X, G, \psi)$ into $\operatorname{GTS}(Y, H, \omega)$ if for any pair $[g, x] \in G \times X$ the equality

$$
\omega(\mu(g), \varphi(x))=\varphi(\psi(g, x))
$$

is satisfied, i.e. the diagram, where $\varphi^{*}: \mathcal{P}^{*}(X) \rightarrow \mathcal{P}^{*}(Y)$ is the corresponding liftation of the mapping $\varphi: X \rightarrow Y$,

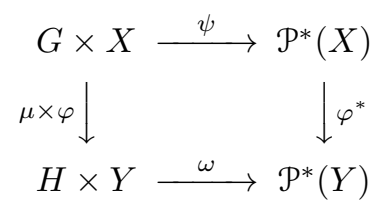

commutes.

Example 4.10. Let $X, Y$ be equivalent non-empty sets and $f: X \rightarrow X$, $h: Y \rightarrow Y$ be mappings such that mono-unary algebras $(X, f) \cong(Y, h)$. Denote $G=\left\{f^{n} ; n \in \mathbb{N}_{0}\right\}, H=\left\{h^{n}, n \in \mathbb{N}_{0}\right\}$ and define binary hyperoperations $\star: G \times G \rightarrow \mathcal{P}^{*}(G)$, and $\bullet: H \times H \rightarrow \mathcal{P}^{*}(H)$, by

$f^{n} \star f^{m}=\left\{f^{k} ; k \in \mathbb{N}_{0}, m+n \leq k\right\}$ and $h^{n} \bullet h^{m}=\left\{h^{k} ; k \in \mathbb{N}_{0}, m+n \leq k\right\}$.

Define mappings $\psi: G \times X \rightarrow \mathcal{P}^{*}(X), \omega: H \times Y \rightarrow \mathcal{P}^{*}(Y)$, by the same rule $\psi\left(f^{n}, x\right)=\left\{f^{k}(x) ; k \in\{0, n, n+1, n+2, \ldots\}\right\}, \omega\left(h^{n}, y\right)=\left\{h^{k}(y) ; k \in\right.$ $\{0, n, n+1, n+2, \ldots\}\}$. Suppose $\xi:(X, f) \rightarrow(Y, h)$ is an isomorphism and $\varphi:(X, f) \rightarrow(Y, h)$ a homomorphism of the mono-unary algebra $(X, f)$ onto the mono-unary algebra $(Y, h)$. Denote $\Phi=[\mu, \varphi]$ the pair of mappings such that $\mu\left(f^{n}\right)=\xi \circ f^{n} \circ \xi^{-1}$. Then $\Phi$ is a homomorphism of the generalized transformation semihypergroup $(X, G, \psi)$ into the $\operatorname{GTS}(Y, H, \omega)$. 
Indeed, for an arbitrary pair $\left[f^{n}, x\right] \in G \times X$ we have

$$
\begin{aligned}
\varphi\left(\psi\left(f^{n}, x\right)\right) & =\varphi\left\{x, f^{n}(x), f^{n+1}(x), \ldots\right\}=\left\{\varphi(x), \varphi\left(f^{n}(x)\right), \varphi\left(f^{n+1}(x)\right), \ldots\right\} \\
& =\left\{\varphi(x), \varphi\left(h^{n}(x)\right), \varphi\left(h^{n+1}(x)\right), \ldots\right\}=\omega\left(h^{n}, \varphi(x)\right) \\
& \left.=\omega\left(\xi \circ f^{n} \circ \xi^{-1}, \varphi(x)\right)=\omega\left(\mu\left(f^{n}\right), \varphi(x)\right)=\omega(\mu \times \varphi)\left[f^{n}, x\right]\right) .
\end{aligned}
$$

The following example of generalized transformation hypergroup is based on consideration published in [1].

Example 4.11. Let $J \subset \mathbb{R}$ be an open interval and denote $C^{\infty}(J)$ the ring of all infinitely differentiable functions on J. Let us consider the set $\mathbb{L} \mathbb{A}_{n}(J)$, $n \in \mathbb{N}$, of linear differential operators of the $\mathrm{n}$-th order in the form

$$
L\left(p_{0}, \ldots, p_{n-1}\right)=\frac{d^{n}}{d x^{n}}+\sum_{k=0}^{n-1} p_{k}(x) \frac{d^{k}}{d x^{k}} .
$$

Where $p_{k} \in C^{\infty}(J), k=0,1, \ldots, n-1 ; L\left(p_{0}, \ldots, p_{n-1}\right): C^{\infty}(J) \longrightarrow C^{\infty}(J)$, thus

$L\left(p_{0}, \ldots, p_{n-1}\right)(f)=f^{(n)}(x)+p_{n-1}(x) f^{(n-1)}(x)+\cdots+p_{0}(x) f(x), f \in C^{\infty}(J)$.

Let $\delta_{i j}$ stand for the Kronecker symbol $\delta$. For any but fixed $m \in\{0,1, \ldots, n-1\}$ we denote by

$$
\mathbb{L A}_{n}(J)_{m}=\left\{L\left(p_{0}, \ldots, p_{n-1}\right) \mid p_{k} \in C^{\infty}(J), p_{m}>0\right\} .
$$

Shortly we put $\mathbf{p}=\left(p_{0}(x), \ldots, p_{n-1}(x)\right), x \in J$ and on the set $\mathbb{L} \mathbb{A}_{n}(J)_{m}$ we define a binary operation " ${ }_{m}$ " and a binary relation $\leq_{m}$ in this way:

$$
L(\mathbf{p}) \circ_{m} L(\mathbf{q})=L(\mathbf{u})
$$

where $u_{k}(x)=p_{m}(x) q_{k}(x)+\left(1-\delta_{k m}\right) p_{k}(x), x \in J, 0 \leq k \leq n-1$, and

$$
L(\mathbf{p}) \leq_{m} L(\mathbf{q})
$$

whenever $p_{k}(x) \leq q_{k}(x), k \neq m, k \in\{0,1, \ldots, n-1\}, p_{m}(x)=q_{m}(x), x \in J$.

It is easy to verify that $\left(, \circ_{m}, \leq_{m}\right)$ is an ordered noncommutative group with the neutral element $D(\mathbf{w})$, where $D(\mathbf{w})=\left(w_{0}, \ldots, w_{n-1}\right), w_{k}(x)=\delta_{k m}$. An inverse to any $D(\mathbf{q})$ is $D^{-1}(\mathbf{q})=\left(\frac{-q_{0}}{q_{m}}, \ldots, \frac{1}{q_{m}}, \ldots, \frac{-q_{n-1}}{q_{m}}\right)$.

Let $(\mathbb{Z},+, \leq)$ be the additive group of all integers with the usual ordering " $\leq$ ". Then by Lemma 2.1 the structure $(\mathbb{Z}, \star)$, where $\star: \mathbb{Z} \times \mathbb{Z} \longrightarrow \mathcal{P}^{*}(\mathbb{Z})$ was defined by $k \star l=[k+l)_{\leq}$is a hypergroup. 
For fixed $D(\mathbf{q}) \in \mathbb{L} \mathbb{A}_{n}(J)_{m}$ we define an action $\psi_{q}: \mathbb{Z} \times \mathbb{L} \mathbb{A}_{n}(J)_{m} \longrightarrow$ $\mathcal{P}^{*}\left(\mathbb{L A}_{n}(J)_{m}\right)$ as follows,

$$
\psi_{q}(k, L(\mathbf{p}))=\left\{L^{t}(\mathbf{q}) \circ_{m} L(\mathbf{p}) \mid t \leq k\right\} .
$$

So $\left(\mathbb{L A}_{n}(J)_{m}, \mathbb{Z}, \varphi_{q}\right)$ is a generalized transformation hypergroup.

\subsection{L-hyperaction of a non-empty set on a hyperstructure}

Let us define another type of action of a set on a hyperstructure (a new definition).

Definition 4.12. Let $(G, \star)$ be a hyperstructure and $X$ be a non-empty set. A generalized action of $X$ on $\mathrm{G}$ is a map $\psi: X \times G \longrightarrow \mathcal{P}^{*}(G)$ defined by $\psi(g \star h, x)=\psi(g, x) \star \psi(h, x)$.

Example 4.13. Let $X$ be a set and $P$ be a polygroup. Then $X$ is a set of hyperoperators on $P$ and $P$ is a $X$-polygroup (polygroup with hyperoperators) if there is a map $\psi: X \times P \longrightarrow \mathcal{P}^{*}(P)$ denoted by $(x, g) \longrightarrow x g$, such that $x(g h)=(x g)(x h)$ for all $x \in X$ and $g, h \in P$.

For more details we refer to [7].

Proposition 4.14. A hypergroupoid $(H, \star)$ is a quasi-order hypergroup if and only if there exist quasi-order relation $\rho$ on the set $H$ such that for all $(a, b) \in$ $H \times H$ there is $a \star b=\rho(a) \cup \rho(b)$ where $\rho(a)=\{x \in H \mid a \rho x\}$.

For the proof see e.g. [4], pages 96-97.

\section{Some applications}

In fact, we shall generalize some results of $[6,7]$ by considering our definitions. All the objects considered are assumed to be of class $C^{\infty}$ and differential forms will take their values in the field of complex numbers.

A Fredholm-Volterra integral operator, where $J=(a, b)$, can be written as follows:

$$
\begin{aligned}
F(\lambda, \mu, K, L, f): C(J \times[0,+\infty)) & \longrightarrow C(J \times[0,+\infty)) \\
F(\lambda, \mu, K(x, t, s), L(x, t, \tau), f(x, t))(\varphi(x, t)) & =\lambda \int_{a}^{b} K(x, t, s) \varphi(s, t) \mathrm{d} s \\
& +\mu \int_{0}^{t} L(x, t, \tau) \varphi(x, \tau) d \tau+f(x, t)
\end{aligned}
$$


Kernels $K(x, t, s) \in C(J \times[0,+\infty) \times J)$ and $L(x, t, \tau) \in C(J \times[0,+\infty),[0, t])$, are real or complex valued functions (mostly positive real functions). $f(x, t) \in$ $C(J, \times[0,+\infty))$ and $\lambda, \mu$ are two real numerical parameters.

Usually there are considered Fredholm-Volterra integral equations with a nondegenerate Lebesgue square integrable kernels $K(x, t, s)$ and $L(x, t, \tau)$. In this contribution we will construct hyperstructures on the set of operators $F(\lambda, \mu, K(x, t, s), L(x, t, \tau), f(x, t))$ with continuous functions $K, L, f$ and two nonzero parameters $\lambda, \mu$. For our purposes we will consider continuous positive functions only, in order to avoid some obstacles with integrability of functions in the form of fractions.

Let us denote by $F V=\{F(\lambda, \mu, K(x, t, s), L(x, t, \tau), f(x, t)) \mid \lambda, \mu \in \mathbb{R}$, $\lambda^{2}+\mu^{2} \neq 0, K(x, t, s) \in C(J \times[0,+\infty) \times J), L(x, t, \tau) \in C(J \times[0,+\infty),[0, t])$, $f(x, t) \in C(J, \times[0,+\infty))\}$.

For any pairs of operators $F\left(\lambda_{1}, \mu_{1}, K_{1}, L_{1}, f_{1}\right), F\left(\lambda_{2}, \mu_{2}, K_{2}, L_{2}, f_{2}\right)$ in $F V$ let us define a binary operation "o"

$$
\begin{aligned}
& F\left(\lambda_{1}, \mu_{1}, K_{1}, L_{1}, f_{1}\right) \circ F\left(\lambda_{2}, \mu_{2}, K_{2}, L_{2}, f_{2}\right)= \\
& \quad F\left(\lambda_{1} \lambda_{2}, \mu_{1} \mu_{2}, K_{2} f_{1}+K_{1}, L_{2} f_{1}+L_{1}, f_{1} f_{2}\right)
\end{aligned}
$$

and a binary relation " $\leq$ "

$$
\begin{gathered}
F\left(\lambda_{1}, \mu_{1}, K_{1}, L_{1}, f_{1}\right) \leq F\left(\lambda_{2}, \mu_{2}, K_{2}, L_{2}, f_{2}\right) \\
\text { if and only if } \\
\lambda_{1}=\lambda_{2}, \mu_{1}=\mu_{2}, f_{1}(x, t) \equiv f_{2}(x, t)
\end{gathered}
$$

for any $(x, t) \in(J \times[0, \infty)), K_{1}(x, t, s) \leq K_{2}(x, t, s)$, for any $(x, t, s) \in(J \times$ $[0,+\infty) \times J)$ and $L_{1}(x, t, \tau) \leq L_{2}(x, t, \tau)$ for any $(x, t, \tau) \in(J \times[0,+\infty),[0, t])$. From the previous it is clear that the following proposition holds.

Proposition 5.1. The triple $(F V, \circ, \leq)$ is a noncommutative ordered group.

Now we apply the simple construction of a hypergroup from Lemma 2.1 onto this considered concrete case of integral operators.

For an arbitrary pair of operators $F\left(\lambda_{1}, \mu_{1}, K_{1}, L_{1}, f_{1}\right), F\left(\lambda_{2}, \mu_{2}, K_{2}, L_{2}, f_{2}\right) \in$ $F V$ we define a hyperoperation $\star: F V \times F V \longrightarrow \mathcal{P}^{*}(F V)$ as follows:

$$
\begin{aligned}
& F\left(\lambda_{1}, \mu_{1}, K_{1}, L_{1}, f_{1}\right) \star F\left(\lambda_{2}, \mu_{2}, K_{2}, L_{2}, f_{2}\right) \\
& =\left\{F(\lambda, \mu, K, L, f) \in F V \mid F\left(\lambda_{1}, \mu_{1}, K_{1}, L_{1}, f_{1}\right) \circ F\left(\lambda_{2}, \mu_{2}, K_{2}, L_{2}, f_{2}\right)\right. \\
& \leq F(\lambda, \mu, K, L, f)\} \\
& =\left\{F(\lambda, \mu, K, L, f) \in F V \mid \lambda=\lambda_{1} \lambda_{2}, \mu=\mu_{1} \mu_{2}, f=f_{1} f_{2},\right. \\
& \left.K_{2} f_{1}+K_{1} \leq K, L_{2} f_{1}+L_{1} \leq L\right\} .
\end{aligned}
$$

In a similar way as in [11] we obtain the following assertion. 
Proposition 5.2. The pair $(F V, \star)$ is a noncommutative transposition hypergroup.

If in the Fredholm-Volterra operators $\mu=0, L=0$ then Fredholm-Volterra operators coincide to Fredholm operators (see [10]) and hypergroup $(F V, \star)$ denote by $(\mathcal{F}, \star)$. By a centralizer of an element a of the group $G$ we mean as usual its subgroup $C_{G}(a)=\{x \in G \mid a x=x a\}$. A centralizer of an element $F(\lambda, K, f) \in \mathbf{F}$ is a subgroup $C_{\mathbf{F}}(F(\lambda, K, f))=\{F(\mu, L, g) \in \mathbf{F} \mid F(\mu, L, g) \circ$ $F(\lambda, K, f)=F(\lambda, K, f) \circ F(\mu, L, g)\}$ for any pair $(x, s) \in J \times J$.

Let $C_{\mathbf{F}}\left(F\left(\lambda_{0}, K_{0}, f_{0}\right)\right)=C_{\mathbf{F}}$ the centralizer of the operator $F\left(\lambda_{0}, K_{0}, f_{0}\right)$ within the group $(\mathbf{F}, \circ)$. Let us define a hyperoperation $\circledast: C_{\mathbf{F}} \times C_{\mathbf{F}} \longrightarrow$ $\mathcal{P}^{*}\left(C_{\mathbf{F}}\right)$ as follows:

$$
\begin{aligned}
F\left(\lambda_{1}, K_{1}, f_{1}\right) & \circledast F\left(\lambda_{2}, K_{2}, f_{2}\right)= \\
= & \left\{F^{n}\left(\lambda_{0}, K_{0}, f_{0}\right) \circ F\left(\lambda_{2}, K_{2}, f_{2}\right) \circ F\left(\lambda_{1}, K_{1}, f_{1}\right) \mid n \in \mathbb{N}_{0}\right\} .
\end{aligned}
$$

Š. Hošková and J. Chvalina in Proposition 3.5 of [11] affirmed that the system

$$
\mathcal{M}\left(F\left(\lambda_{0}, K_{0}, f_{0}\right)\right)=\left(C(J),\left(C_{\mathbf{F}}, \star\right), \delta\right)
$$

where the mapping $\delta: C_{\mathbf{F}} \times C(J) \longrightarrow C(J)$ is defined by

$$
\delta(F(\lambda, K, f), \varphi)=(F(\lambda, K, f) \circ F(\lambda, K, f))(\varphi(x))
$$

is a transformation semihypergroup with the phase set $C(J)$ and the phase semihypergroup $\left(C_{\mathbf{F}}, \star\right)$.

In the following counterexample we show that GMAC is not valid.

Example 5.3. Let $F\left(\lambda_{0}, K_{0}, f_{0}\right)=F(1, x, 2) \in C_{\mathbf{F}}$ and $F\left(\lambda_{1}, K_{1}, f_{1}\right)=$ $F\left(1, x^{3}, x^{2}+1\right), F\left(\lambda_{2}, K_{2}, f_{2}\right)=F\left(1, x^{2}, x+1\right)$ arbitrary element in $C_{\mathbf{F}}$ and $\varphi(x)=x$. It is easy to see that

$\delta\left(F\left(1, x^{2}, x+1\right), \delta\left(F\left(1, x^{3}, x^{2}+1\right), x\right)\right) \notin \delta\left(F\left(1, x^{3}, x^{2}+1\right) \circledast F\left(1, x^{2}, x+1\right), x\right)$.

Therefore, it is easy to see that the condition (GMAC) in Proposition 3.5 of [11] is not valid.

But, if we put $T=\mathcal{F} \times C(J)$ and $\mathcal{M}\left(F\left(\lambda_{0}, K_{0}, f_{0}\right)\right)=\left(T,\left(C_{\mathbf{F}}, \star\right), \pi\right)$ such that $\pi: C_{\mathbf{F}} \times T \longrightarrow \mathcal{P}^{*}(T)$ is defined by

$$
\begin{aligned}
& \pi\left(F\left(\lambda_{1}, K_{1}, f_{1}\right),(F(\lambda, K, f), \varphi)\right)= \\
& \quad=\left(F\left(\lambda_{0}, K_{0}, f_{0}\right) \circ F\left(\lambda_{1}, K_{1}, f_{1}\right) \circ F(\lambda, K, f), \varphi\right) .
\end{aligned}
$$

So the GMAG is satisfied and we have: 
Proposition 5.4. The system $\mathcal{M}\left(F\left(\lambda_{0}, K_{0}, f_{0}\right)\right)$ is a discrete transformation hypergroup with the phase set $T$ and phase semihypergroup $\left(C_{\mathbf{F}}, \star\right)$.

Proof. Suppose $F_{1}, F_{2} \in \mathbb{C}_{F}, F \in \mathcal{F}$ and $\varphi \in \mathbb{C}(J)$. Then $\pi\left(F_{1}, \pi\left(F_{2},[F, \varphi]\right)\right)$ $=\pi\left(F_{1},\left[F_{0} \circ F_{2} \circ F, \varphi\right]\right)=\left[F_{0} \circ F_{1} \circ F_{0} \circ F_{2} \circ F, \varphi\right]=\left[F_{0}^{2} \circ F_{1} \circ F_{2} \circ F, \varphi\right]$. Denote by $\psi=F_{0} \circ F_{1} \circ F_{2}$. Since $\psi \in\left\{F_{0}^{n} \circ F_{1} \circ F_{2} ; n \in \mathbb{N}\right\}$, we have $\left[F_{0}^{2} \circ F_{1} \circ F_{2} \circ F, \varphi\right]=\left[F_{0} \circ \psi \circ F, \varphi\right]=\pi(\psi,[F, \varphi]) \in\{\pi(\Phi,[F, \varphi])\} ; \Phi \in\left\{F_{0}^{n} \circ\right.$ $\left.F_{1} \circ F_{2} ; n \in \mathbb{N}\right\}=\left\{\pi(\Phi,[F, \varphi]) ; \Phi \in F_{1} \star F_{2}\right\}=\pi\left(F_{1} \star F_{2},[F, \varphi]\right)$, consequently $\pi\left(F_{1}, \pi\left(F_{2},[F, \varphi]\right)\right) \in \pi\left(F_{1} \star F_{2},[F, \varphi]\right)$, hence GMAC is satisfied.

Let us calculate the counterexample 5.3 in more details.

$$
\begin{aligned}
& F_{0}=F\left(\lambda_{0}, K_{0}, F_{0}\right)=F(1, x, 2), \\
& F_{1}=F\left(\lambda_{1}, K_{1}, F_{1}\right)=F\left(1, x^{3}, x^{2}+1\right), \\
& F_{2}=F\left(\lambda_{2}, K_{2}, F_{2}\right)=F\left(1, x^{2}, x+1\right) .
\end{aligned}
$$

Let $\langle a, b\rangle=<0,1>$ and $\varphi(x)=x$ so, for the left hand side of the relation

$$
\delta\left(\delta\left(\varphi, F_{1}\right), F_{2}\right) \in \delta\left(\varphi, F_{1} \star F_{2}\right)
$$

we get:

$$
\begin{aligned}
& \delta\left(\varphi, F_{1}\right)=F\left(\lambda_{0} \lambda_{1},\right.\left.K_{1} f_{0}+K_{0}, f_{0} f_{1}\right)(\varphi(x))=\int_{0}^{1}\left(2 x^{3}+x\right) s \mathrm{~d} s+2 x^{2}+2 \\
&=x^{3}+2 x^{2}+\frac{x}{2}+2=\psi(x) . \text { So, we have } \\
& \delta\left(\delta\left(\varphi, F_{1}\right), F_{2}\right)=F\left(\lambda_{0} \lambda_{2}, K_{2} f_{0}+K_{0}, f_{0} f_{2}\right)(\psi(x)) \\
&=\int_{0}^{1}\left(2 x^{2}+x\right)\left(s^{3}+2 s^{2}+\frac{s}{2}+2\right) \mathrm{d} s+2 x+2 \\
&=\frac{19}{6}\left(2 x^{2}+x\right)+2 x+2=\frac{19}{3} x^{2}+\frac{31}{6} x+2=q(x) .
\end{aligned}
$$

To calculate the right hand side of the relation (5.1) we need at first:

$F_{1} \star F_{2}=\left\{F_{0}^{n} \cdot F_{2} \cdot F_{1} ; n \in \mathbb{N}_{0}\right\}$,

$F_{1} \cdot F_{2}=F\left(\lambda_{1} \lambda_{2}, K_{2} f_{1}+K_{1}, f_{1} f_{2}\right)$,

$F_{2} \cdot F_{1}=F\left(\lambda_{1} \lambda_{2}, K_{1} f_{2}+K_{2}, f_{1} f_{2}\right)$,

$F_{0} \cdot F_{0}=F\left(\lambda_{0}^{2}, K_{0} f_{0}+K_{0}, f_{0}^{2}\right)=F\left(\lambda_{0}^{2}, K_{0}\left(f_{0}+1\right), f_{0}^{2}\right)=F_{0}^{2}$,

$F_{0}^{3}=F_{0}^{2} \cdot F_{0}=F\left(\lambda_{0}^{3}, K_{0} f_{0}^{2}+K_{0}\left(f_{0}+1\right), f_{0}^{3}\right)=F\left(\lambda_{0}^{3}, K_{0}\left(f_{0}^{2}+f_{0}+1\right), f_{0}^{3}\right)$, 


$$
F_{0}^{n}=F\left(\lambda_{0}^{n}, K_{0}\left(f_{0}^{n-1}+f_{0}^{n-2}+\cdots+f_{0}+1\right), f_{0}^{n}\right)=F\left(\lambda_{0}^{n}, K_{0} \frac{f_{0}^{n}-1}{f_{0}-1}, f_{0}^{n}\right) \text {, if }
$$

$f_{0} \neq 1$. For functions chosen in the counterexample we get:

$$
\begin{aligned}
F_{0}^{n} & =F\left(1,\left(2^{n}-1\right) x, 2^{n}\right) . \\
F_{2} \cdot F_{1} & =F\left(1, x^{3}(x+1),\left(x^{2}+1\right)(x+1),\right. \\
F_{0}^{n} \cdot F_{2} \cdot F_{1} & =F\left(1,2^{n} \cdot x^{3}(x+1)+\left(2^{n}-1\right) x, 2^{n}\left(x^{2}+1\right)(x+1)\right) \\
& =F\left(1,2^{n} x^{4}+2^{n} x^{3}+\left(2^{n}-1\right) x, 2^{n} x^{3}+2^{n} x+2^{n} x^{2}+2^{n}\right) .
\end{aligned}
$$

Thus,

$$
\begin{aligned}
& \left(\varphi, F_{0}^{n} \cdot F_{2} \cdot F_{1}\right)=\left(F_{0} \cdot F_{0}^{n} \cdot F_{2} \cdot F_{1}\right)(\varphi(x))\left(F_{0}^{n+1} \cdot F_{2} \cdot F_{1}\right)(\varphi(x)) \\
& \quad=F\left(1,2^{n+1} x^{4}+2^{n+1} x^{3}+\left(2^{n+1}-1\right) x, 2^{n+1} x^{3}+2^{n+1} x+2^{n+1} x^{2}+2^{n+1}\right) \\
& \quad=\int_{0}^{1}\left[2^{n+1}\left(x^{4}+x^{3}+x\right)-x\right] s \mathrm{~d} s+2^{n+1} x^{3}+2^{n+1} x+2^{n+1} x^{2}+2^{n+1} \\
& \quad=2^{n} x^{4}+3 \cdot 2^{n} x^{3}+\left(3 \cdot 2^{n}-\frac{1}{2}\right) x+2^{n+1} x^{2}+2^{n+1}=P_{n}(x) .
\end{aligned}
$$

So for the right hand side of the relation (5.1) we get $\delta\left(\varphi, F_{1} \star F_{2}\right)=\left\{P_{n}(x) ; n \in\right.$ $\left.\mathbb{N}_{0}\right\}$. Denote by st $P$ the power of a polynomial $P$, st $P_{n}(x)=4$ for any $n \in N_{0}$, whereas st $q(x)=2$, hence $q \neq \delta\left(\varphi, F_{1} \star F_{2}\right)$.

The Proposition 5.4 is not the only chance how to correct the mistake done in Proposition 3.5 in [11]. Š. Hošková and J. Chvalina have already published one way how to correct it in [12]. For the availability and reader's convenience we present here the most important part of it. We shall consider smooth functions, i.e., $u \in \mathbf{C}^{\infty}(\Omega)$. Let $\mathrm{P}\left(a_{1}, \ldots, a_{n}, p\right): \mathbf{C}^{\infty}(\Omega) \rightarrow \mathbf{C}^{\infty}(\Omega)$ be a fixed chosen operator,

$$
\mathrm{P}\left(a_{1}, \ldots, a_{n}, p\right) u=\sum_{k=1}^{n} a_{k}\left(x_{1}, \ldots, x_{n}\right) \frac{\partial u}{\partial x_{k}}+p\left(x_{1}, \ldots, x_{n}\right) u\left(x_{1}, \ldots, x_{n}\right) .
$$

Denote by $\mathrm{Ct}(\mathrm{P})$ the set of all differential operators $\mathrm{D} \in \mathbb{L}^{1} \mathbb{D}(\Omega)$ commuting with the operator $\mathrm{P}$, i.e.,

$$
\mathrm{Ct}(\mathrm{P})=\left\{\mathrm{D} \in \mathbb{L}^{1} \mathbb{D}(\Omega) ; \mathrm{P} \cdot \mathrm{D}=\mathrm{D} \cdot \mathrm{P}\right\} .
$$

Since the identity operator Id belongs to $\mathrm{Ct}(\mathrm{P})$, this set endowed with the unique operation "." is a monoid which is called the centralizer of the operator P within the group $\left(\mathbb{L}^{1} \mathbb{D}(\Omega), \cdot\right)$.

Lemma 5.5. ([12]) Operators $\mathrm{D}_{1}=\mathrm{D}\left(a_{1}, \ldots, a_{n}, p\right), \mathrm{D}_{2}=\mathrm{D}\left(b_{1}, \ldots, b_{n}, q\right)$ from the group $\mathbb{L}^{1} \mathbb{D}(\Omega)$ are commuting if and if for any $k=1,2, \ldots, n$ and 
any point $\left[x_{1}, \ldots, x_{n}\right] \in \Omega$ there holds

$$
\left|\begin{array}{cc}
1-p\left(x_{1}, \ldots, x_{n}\right) & 1-q\left(x_{1}, \ldots, x_{n}\right) \\
a_{k}\left(x_{1}, \ldots, x_{n}\right) & b_{k}\left(x_{1}, \ldots, x_{n}\right)
\end{array}\right|=0 .
$$

Now, for any pair $\mathrm{D}_{\alpha}, \mathrm{D}_{\beta} \in \mathrm{Ct}(\mathrm{P})$ define a hyperoperation " $\odot$ " as follows:

$$
\odot: \mathrm{Ct}(\mathrm{P}) \times \mathrm{Ct}(\mathrm{P}) \rightarrow \mathcal{P}^{*}(\mathrm{Ct}(\mathrm{P}))
$$

by

$$
\mathrm{D}_{\alpha} \odot \mathrm{D}_{\beta}=\left\{\mathrm{P}^{n} \cdot \mathrm{D}_{\beta} \cdot \mathrm{D}_{\alpha} ; n \in \mathbb{N}\right\} .
$$

Consider the binary relation $\rho_{\mathrm{P}} \subset \mathrm{Ct}(\mathrm{P}) \times \mathrm{Ct}(\mathrm{P})$ defined by

$$
\mathrm{D}_{\alpha} \rho_{\mathrm{P}} \mathrm{D}_{\beta} \text { if and only if } \mathrm{D}_{\beta}=\mathrm{P}^{n} \cdot \mathrm{D}_{\alpha}
$$

for some $n \in \mathbb{N}_{0}$. We get without any effort (see [12]) that $\left(\mathrm{Ct}(\mathrm{P}), \cdot, \rho_{\mathrm{P}}\right)$ is a quasi-ordered monoid.

Further, $\mathrm{D}_{\alpha} \odot \mathrm{D}_{\beta}=\rho_{\mathrm{P}}\left(\mathrm{D}_{\beta} \cdot \mathrm{D}_{\alpha}\right)=\left[\mathrm{D}_{\beta} \cdot \mathrm{D}_{\alpha}\right)_{\rho_{\mathrm{P}}}$ and by 2.1 we obtain that $(\mathrm{Ct}(\mathrm{P}), \odot)$ is a hypergroup (noncommutative, in general).

As usually $(\mathrm{Ct}(\mathrm{P}))^{+}$with the operation of concatenation means the free semigroup of finite non-empty words formed by operators from the set $\mathrm{Ct}(\mathrm{P})$.

Denote $S_{\mathrm{P}}=\left\{\left(\mathrm{P} \cdot \mathrm{D}_{1} \cdots \cdots \mathrm{D}_{n}\right)(f) ; f \in \mathbf{C}^{\infty}(\Omega), \mathrm{D}_{1} \cdots \mathrm{D}_{n} \in(\mathrm{Ct}(\mathrm{P}))^{+}\right\}$ and $\mathbb{M}\left(S_{\mathrm{P}}\right)$ the triple $\left(S_{\mathrm{P}},(\mathrm{Ct}(\mathrm{P}), \odot), \delta_{\mathrm{P}}\right)$, where the action or transition function $\delta_{\mathrm{P}}: S_{\mathrm{P}} \times \mathrm{Ct}(\mathrm{P}) \rightarrow S_{\mathrm{P}}$ is defined by the rule

$$
\delta_{\mathrm{P}}\left(\left(\mathrm{P} \cdot \mathrm{D}_{1} \cdots \cdots \mathrm{D}_{n}\right)(f), \mathrm{D}_{\alpha}\right)=\left(\mathrm{P} \cdot \mathrm{D}_{\alpha} \cdot \mathrm{D}_{1} \cdots \cdots \mathrm{D}_{n}\right)(f)
$$

for any function $f \in \mathbf{C}^{\infty}(\Omega)$ and any operator $\mathrm{D}_{\alpha} \in \mathrm{Ct}(\mathrm{P})$. The transition function $\delta_{\mathrm{P}}$ satisfies the Generalized Mixed Associativity Condition.

Indeed, suppose $f \in \mathbf{C}^{\infty}(\Omega), \mathrm{D}_{\alpha}, \mathrm{D}_{\beta}, \mathrm{D}_{1}, \mathrm{D}_{2} \in \mathrm{Ct}(\mathrm{P})$ are arbitrary elements. We have

$$
\begin{aligned}
& \delta_{\mathrm{P}}\left(\delta_{\mathrm{P}}\left(\left(\mathrm{P} \cdot \mathrm{D}_{1} \cdots \mathrm{D}_{n}\right)(f), \mathrm{D}_{\alpha}\right), \mathrm{D}_{\beta}\right)=\delta_{\mathrm{P}}\left(\left(\mathrm{P} \cdot \mathrm{D}_{\alpha} \cdot \mathrm{D}_{1} \cdots \mathrm{D}_{n}\right)(f), \mathrm{D}_{\beta}\right) \\
& =\left(\left(\mathrm{P} \cdot \mathrm{D}_{\beta} \cdot \mathrm{D}_{\alpha} \cdot \mathrm{D}_{1} \cdots \cdots \mathrm{D}_{n}\right)(f)\right) \in\left\{\left(\mathrm{P}^{n+1} \cdot \mathrm{D}_{\beta} \cdot \mathrm{D}_{\alpha} \cdot \mathrm{D}_{1} \cdots \mathrm{D}_{n}\right)(f), n \in \mathbb{N}_{0}\right\} \\
& =\delta_{\mathrm{P}}\left(\left(\mathrm{P} \cdot \mathrm{D}_{1} \cdots \cdots \mathrm{D}_{n}\right)(f), \mathrm{P}^{n} \cdot \mathrm{D}_{\alpha} \cdot \mathrm{D}_{\beta}\right)=\delta_{\mathrm{P}}\left(\left(\mathrm{P} \cdot \mathrm{D}_{1} \cdots \mathrm{D}_{n}\right)(f), \mathrm{D}_{\alpha} \odot \mathrm{D}_{\beta}\right),
\end{aligned}
$$

so GMAC is satisfied, i.e., the triple $\mathbb{M}\left(S_{\mathrm{P}}\right)=\left(S_{\mathrm{P}},(\mathrm{Ct}(\mathrm{P}), \odot), \delta_{\mathrm{P}}\right)$ is a multiautomaton.

Using the above considerations concerning the centralizer hypergroup of partial differential operators we obtain the second possible correction of the 
wrong Proposition 3.5 of [11]. So, consider the above described centralizer hypergroup $\left(C_{F}, \circledast\right)$ of Fredholm integral operators (defined above- after Proposition 5.2. Denote by $C_{F}^{+}$the free semigroup of finite non-empty words formed by operators from the set $C_{F}$ with binary operation of concatenation. We denote

$$
S_{F}=\left\{\left(F \circ F_{1} \circ \cdots \circ F_{n}\right)(\varphi) ; \varphi \in \mathbb{C}(J), F_{k} \in C_{F}^{+}, k=1,2 \ldots, n\right\} .
$$

Define a function $\delta_{F}: S_{F} \times C_{F} \rightarrow S_{F}$ by the rule

$$
\left.\delta_{F}\left(F \circ F_{1} \circ \cdots \circ F_{n}\right)(\varphi), G\right)=\left(F \circ G \circ F_{1} \circ \cdots \circ F_{n}\right)(\varphi)
$$

for any function $\varphi \in \mathbb{C}(J)$ and any integral operator $G \in C_{F}$. In the same way as above we obtain that the triad $\left(S_{F},\left(C_{F}, \circledast\right), \delta_{F}\right)$ is a multiautomaton, i.e. the action of the phase hypergroup $\left(C_{F}, \circledast\right)$ on the state (phase) set $S_{F}$.

\section{Conclusion}

More than 75 years have elapsed since Marty's pioneer paper [17] when the hyperstructure theory was born. During this period the field has experienced an enormous growth. There are applications to geometry, hypergraphs, binary relations, lattices, fuzzy sets and rough sets, automata, cryptography, combinatoric, codes, artificial intelligence, and probability.

Thus, the framework of the hyperstructure theory allows various generalizations of the above mentioned concepts and theories.

In particular, it seems to be interesting to analyse properties of hypergroups of second-order linear differential operators of the Jacobi form creating subhypergroups of hypergroups of more general linear differential operators considered above, or to use hyperstructures for modelling two or more parametrical systems of non-periodic time impulses produced e.g. by foton or neutron illumination generated by disintegration of the Californium 252 .

\section{References}

[1] Chvalina, J., Chvalinová, L., Actions of centralizer hypergroups of $n$-th order linear differential operators on rings of smooth functions, AplimatJ. of Appl. Math. I, No. I, (2008), 45-53.

[2] Chvalina, J., Hošková, Š., Abelizations of weakly associative hyperstructures based on their direct squares, Acta Math. Inform. Univ. Ostraviensis 11, No. 1, (2003), 11-23. 
[3] Chvalina, J., Račková, P., Join spaces of smooth functions and their actions on transposition hypergroups of second order linear differential operators, Aplimat-J. of Appl. Math, I, (2008), 55-63.

[4] Corsini. P., Leoreanu.V., Application of Hyperstructure theory, Kluwer Academic Publishers, (2002).

[5] Cristea, I., Stefanescu, M., Angheluta, C., About the fundamental relations defined on the hypergroupoids associated with binary relations. Eur. J. Comb. 32(1), (2011), 72-81.

[6] Cristea, I., Stefanescu, M., Binary relations and reduced hypergroups. Discrete Math. 308(16), (2008), 3537-3544.

[7] Davvaz, B., Polygroups with hyperoperators, J. of Fuzzy Math. 9, No. 4, (2004), 815-823.

[8] Dehghan Nezhad A., Davvaz, B., Universal Hyperdynamical Systems, Bull. Korean Math. Soc. 47, No.3, (2010), 513-526.

[9] Hashemi, R.S. Applications of transformation hypergroups, M.Sc. Project, written under direction of Dr. Akbar Dehghan Nezhad, Yazd University, Iran, (2008).

[10] Hošková, Š., Chvalina, J., Račková, P., Transposition hypergroups of Fredholm integral operators and related hyperstructures - part 1, Journal of Basic Science, 4, (2008), 43-54.

[11] Hošková, Š., Chvalina, J., Račková, P., Transposition hypergroups of Fredholm integral operators and related hyperstructures - part 2, Journal of Basic Science, 4, (2008), 55-60.

[12] Hošková, Š., Chvalina, J., Action of Hypergroups of the First-order Partial Differential Operators, In: Aplimat 2007, Bratislava, Slovakia, 177185.

[13] Hošková, Š., Chvalina, J., Discrete transformation hypergroup and transformation hypergroups with phase tolerance space, Discrete Math. 308, (2008), 4133-4143.

[14] Hošková, Š., Discrete transformation hypergroups, In: Aplimat 2005, Bratislava, Slovakia, 275-278.

[15] Iwasava, K., On linearly ordered groups, J. Math. Soc. JPN, 1, No.1, (1948), 1-9. 
[16] Jantosciak, J., Transposition hypergroups: Noncommutative join spaces, J. Algebra 187, (1997), 97-19.

[17] Marty, F., Sur une généralisation de la notion de groupe, Huitième Congr. math. Scan. (1934), Stockholm, 45-49.

[18] Mesarovic, M., Takahara, Y., General System Theory, A Math. Foundations; Accad. Press London, (1975).

[19] Novák, M., Potential of the "Ends Lemma" to create ring-like hyperstructures from quasi-ordered(semi)groups, South Bohemia Math. Letters 17, No 1., (2009), 39-50.

[20] Novák, M., Important elements of EL-hyperstructures, Aplimat: 10-th Internat. Conference : STU in Bratislava, 2011, 151-158.

[21] Pelea, C., Identities and multialgebras, Ital. J. Pure Appl. Math., 15, (2004), 83-92.

Jan Chvalina,

Department of Mathematics,

Brno University of Technology,

Technická, 10, 61600 Brno, Czech Republic

Email: chvalina@feec.vutbr.cz

Š́rka Hošková-Mayerová,

Department of Mathematics and Physics,

University of Defence,

Kounicova 65, 66210 Brno, Czech Republic

Email: sarka.mayerova@unob.cz

A. D. Nezhad,

Faculty of Mathematics,

Yazd University, Yazd, Iran

Email: anezhad@yazduni.ac.ir 